\title{
Automatic Status Checks and Recovery for Tundra Microscope
}

\author{
Libor Kubecka $^{1}$, Jan Jisa ${ }^{1}$, Fanis Grollios ${ }^{2}$, Rudolf Siwy ${ }^{1}$, Ondrej Svoboda ${ }^{1}$ and Bart van Knippenberg ${ }^{2}$ \\ ${ }^{1}$ Thermofisher Scientific, Brno, Czech Republic, ${ }^{2}$ Thermo Fisher Scientific, Eindhoven, Netherlands \\ Background
}

The recent popularity of Single Particle Analysis (SPA) has increased the demand for simplified electron microscope (EM) workflows so that scientists can focus on discovering new proteins and protein complex structures instead of learning microscopy [1]. Such democratization requires the microscope to be easy to use. However, the current state of the art in EM still has a lengthy training requirement that can include optical alignment procedures with an understanding of microscope hardware as a prerequisite.

Here, we present our current approach for making the EM workflow less complex and more accessible to inexperienced users by automating those alignments and parameters that do not require user input (Figure 1). This increases the reproducibility of the initial state for data acquisition and increases the number of people that can use the microscope.

Our new automation module tunes the optics and column alignments in a robust, reliable, and user-friendly way. This flattens the learning curve for novice operators and inexperienced users, decreases time-to-data for experienced operators, and ultimately improves overall microscope utility in a multi-user environment.

Traffic lights

A traffic light indicator is designed to report on microscope readiness for SPA acquisition [2]. This indicator monitors microscope HW and alignment status. If the status is sub-optimal, this is indicated by the traffic light, and an automatic recovery procedure is provided to the user. The following functional groups are monitored:

Vacuum:

- Vacuum levels are monitored and an automatic pumping procedure is executed if insufficient.

Source and High Tension:

- FEG and HT conditions are monitored and automatically started and ramped-up if needed.

Camera:

- Temperature and hi-res camera availability are checked. The hi-res camera, Äôs gain corrections are also checked and reacquired if necessary.

Sample loader:

- Monitoring of sample transfer status and cryo-loading station is provided.

Optical parameters: 
- Various optical alignments are periodically checked and corrected if necessary. Among others, the following ones are automated: gun tilt and shift, beam stigmation, beam tilt pivot points, apertures centering, image focusing, astigmatism and coma correction, and eucentric height setting.

\section{Automatic Alignments}

The new Automatic Alignments module groups optical alignments into two sets based on the ability to run with or without a sample. The daily alignments set is designed to run without a sample, once a day, and prepare the microscope to a state suitable for fast sample screening.

Before automated SPA data acquisition, the Dataset alignments set is executed to optimize the optical state of the microscope for the best optical performance. For this purpose, a tiny sample area is used and excluded from data acquisition.

\section{Quality}

To achieve high robustness and performance of the algorithms, all alignments are based on reusable image processing building blocks. These are hierarchically combined into longer alignment sequences. All building blocks and the complete suite of alignment sets are regularly evaluated in our factories. This enables faster and more robust delivery of automation features with every SW release as part of our continuous development.

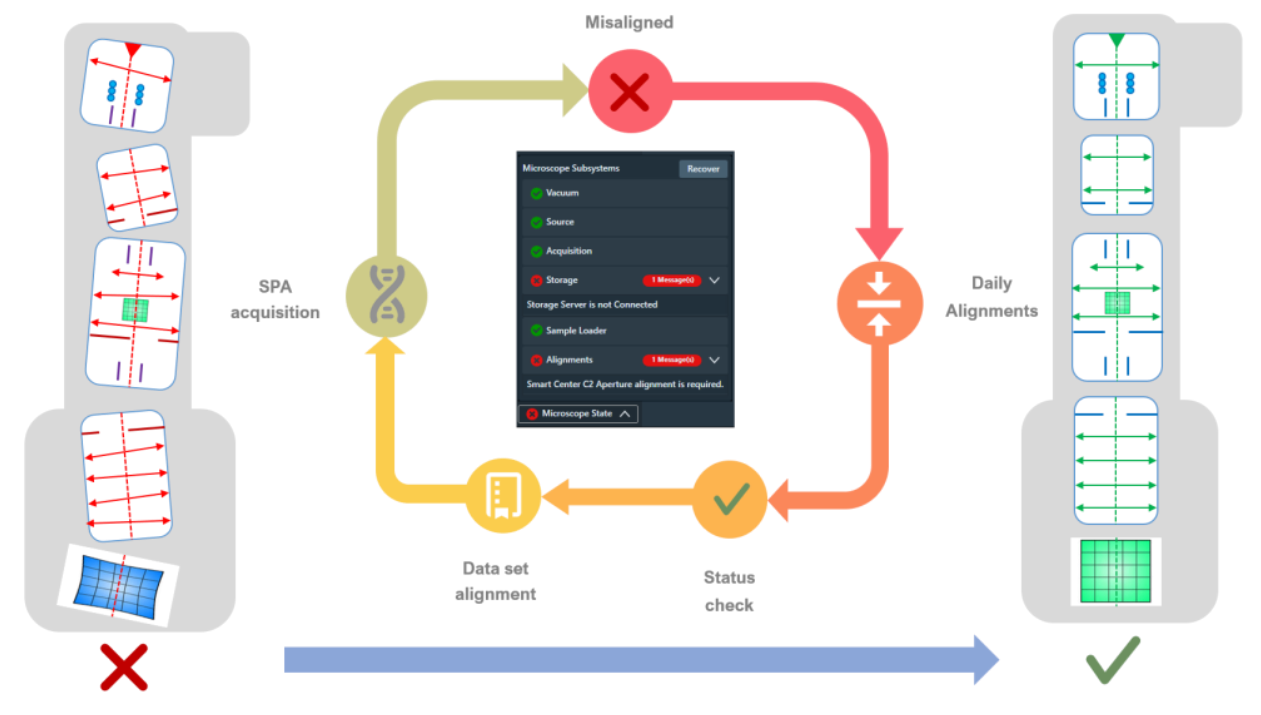

Figure 1. Automatic Microscope Status Checks and Alignments during Daily SPA Routine

\section{References}

[1] Single-particle cryo-EM at atomic resolution, Nakane, T., Kotecha, A., Sente, A. et al. Nature 587, 152$156(2020)$

[2] Thermo Fisher Scientific, EPU 2 Software, last accessed February 2021. https://www.thermofisher.com/nl/en/home/electron-microscopy/products/software-em-3d-vis/epusoftware.html 